iD Tamara Vilhena Teixeira ${ }^{1}$

iD Cleide Braz dos Santos ${ }^{1}$

(iD) Jorginete de Jesus Damião ${ }^{1,2}$

(iD) Luciana Azevedo

Maldonado ${ }^{1,2}$

(iD) Juliana Martins Oliveira ${ }^{2}$

1 Universidade do Estado do Rio de Janeiro, Instituto de Nutrição, Departamento de Nutrição Social. Rio de Janeiro, RJ, Brasil.

2 Universidade do Estado do Rio de Janeiro, Instituto de Nutrição, Núcleo de Alimentação e Nutrição em Políticas Públicas. Rio de Janeiro, RJ, Brasil.

Correspondência Cleide Braz dos Santos cleidebrazqn@yahoo.com.br

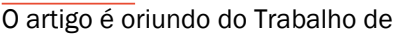
Conclusão de Curso intitulado "Adequação de rótulos de produtos ultraprocessados à legislação de rotulagem para alergênicos: análise de alimentos consumidos por crianças menores de cinco anos usuárias do SUS da cidade do Rio de Janeiro" de Tamara Vilhena Teixeira e Cleide Braz dos Santos, Universidade do Estado do Rio de Janeiro, 2018.

\section{Alimentos ultraprocessados consumidos por crianças atendidas pelo Sistema Único de Saúde na cidade do Rio de Janeiro: análise da adequação dos rótulos à legislação obrigatória para alergênicos}

Ultra-processed foods consumed by children attended by Public Health Care System in the city of Rio de Janeiro: analysis of labels adequacy to required allergen legislation

\section{Resumo}

Objetivo: Analisar os rótulos de alimentos ultraprocessados (AUP) consumidos por crianças menores de cinco anos, quanto à adequação à Resolução n² 26, de 2 de julho de 2015, identificando informações sobre alimentos alergênicos. Métodos: Utilizou-se banco de dados proveniente de pesquisa realizada com amostra representativa da população de pré-escolares usuários da rede básica de saúde no município do Rio de Janeiro ( $n=536$ ). Nesta pesquisa, foram identificados os alimentos ultraprocessados citados nos Recordatórios de 24 horas. Realizou-se o registro fotográfico dos rótulos destes alimentos em pesquisa de mercado. Verificou-se a presença dos alimentos alergênicos e seus derivados na lista de ingredientes dos 303 AUP consumidos, assim como as orientações das informações que deveriam estar presentes no rótulo, à luz da RDC n²6/2015. Resultados: A maior parte $(58,7 \%)$ dos rótulos de alimentos dos grupos de ultraprocessados 
estudados possuía pelo menos um ingrediente alergênico. Foram encontrados 12 ingredientes alergênicos diferentes no grupo dos biscoitos. Dos rótulos analisados, apenas 33,1\% apresentaram mensagem obrigatória para todos os ingredientes alergênicos presentes. Os derivados dos ingredientes alergênicos foram encontrados em 15 grupos de alimentos consumidos pelas crianças. As informações "pode conter" e "contém traços" estavam presentes em 93 rótulos, aproximadamente 30\%. Conclusão: Os achados deste estudo mostram que, no momento da transição da RDC n 26/2015, a maior parte dos rótulos ainda não atendia à legislação.

Palavras-chave: Hipersensibilidade alimentar. Alimentos. Rotulagem de alimentos. Legislação sobre alimentos.

\section{Abstract}

Objective: To analyze the labels of ultra-processed foods (UPF) consumed by children under five years of age, in order to comply with Resolution No. 26 of July 2, 2015, identifying information on allergenic foods. Methods: The database came from a research conducted with a representative sample of the population of pre-school attended at the Public Health Care System in the city of Rio de Janeiro. ( $n=536)$. In this research, the ultra-processed foods were identified from the food listed in the 24-hour dietary recall. A photographic record of the labels of these foods was made during a market research. The presence of allergenic foods and their derivatives was verified in the list of ingredients of the 303 UPFs consumed, as well as the orientations as to the information that should be present on the label, in light of RDC No. 26/2015. Results: Most of the food labels (58.7\%) of the ultra-processed food groups studied had at least one allergenic ingredient. Twelve different allergenic ingredients were found in the cookie group. Of the labels analyzed, only 33.1\% presented the mandatory message showing all the allergenic ingredients present. Derivatives of allergenic ingredients were found in 15 groups of foods consumed by children. The information "may contain" and "contains traces" were present on 93 labels, approximately 30\%. Conclusion: The findings of this study show that, at the time of transition of RDC No. 26/2015, most of the labels still did not meet the legislation.

Keywords: Food hypersensitivity. Food. Food labelling. Legislation on food. 


\section{INTRODUÇÃO}

Alergia alimentar (AA) é o termo reservado às reações imunológicas adversas a alimento ou aditivo alimentar. Essas reações afetam até 10\% da população geral, sendo mais comum em crianças do que em adultos. Estudos internacionais sugerem possível aumento na prevalência de alergias alimentares nos últimos 30 anos. ${ }^{1}$ As manifestações clínicas decorrem da ingestão, inalação ou contato com determinado alimento, que resulta em reações imunológicas. ${ }^{2}$ É documentado que $90 \%$ dos casos de AA são ocasionados por oito alimentos: ovos, leite de vaca, peixes, crustáceos, castanhas, amendoim, trigo e soja. ${ }^{3}$

A oferta de alimentos sólidos antes dos quatro meses de vida, período em que o recémnascido apresenta imaturidade da barreira intestinal, parece elevar o risco de desenvolvimento de AA. Por outro lado, a introdução da alimentação complementar aos seis meses de idade é um fator de proteção para a criança quanto ao risco de desenvolvimento de AA. ${ }^{4}$

Alguns alimentos ultraprocessados (AUP), como macarrão instantâneo, iogurtes, biscoitos recheados, bebidas açucaradas e achocolatados, estão comumente presentes na alimentação complementar de crianças., ${ }^{5,6}$ Dados da Pesquisa Nacional de Demografia e Saúde, referente ao ano de 2006, e da Pesquisa Nacional de Saúde, em 2013, mostram que, respectivamente, 40,5\% das crianças menores de cinco anos consomem refrigerante com frequência e que 60,8\% das crianças menores de dois anos comem biscoitos ou bolachas recheadas. ${ }^{7,8}$

Alimentos ultraprocessados representam risco à saúde de crianças com AA, por contemplarem na lista de ingredientes um conjunto de alimentos alergênicos. Além disso, possuem composição nutricional desbalanceada, visto que comumente são ricos em açúcares e/ou gorduras, sódio, tendem a ser pobres em fibras alimentares e possuem aditivos químicos. ${ }^{9}$

Os cuidadores de crianças que possuem AA procuram por informações sobre os alimentos que serão ofertados, uma vez que a maneira mais eficaz de evitar as reações adversas é a restrição dos alimentos que causam alergias. Por isso, torna-se fundamental que rótulos de alimentos tragam informações claras e legíveis para os consumidores. ${ }^{10}$

O controle e a regulação dos alimentos buscam proteger a população, auxiliando-a na escolha de alimentos que atendam suas necessidades. ${ }^{11}$ Em julho de 2015, foi criada a Resolução de Diretoria Colegiada (RDC) n²6, que dispõe sobre os principais alimentos que causam alergias alimentares. De acordo com esta resolução, os rótulos dos alimentos embalados na ausência do consumidor devem conter informações sobre a natureza alergênica dos ingredientes desses produtos. ${ }^{12}$ 
A fim de garantir a declaração de advertência, além da obrigatoriedade de mensagens quanto à presença intencional de alergênicos e derivados de alergênicos, a resolução também regulamenta declarações sobre a possibilidade de contaminação cruzada, utilizando as frases: "pode conter" ou "contém traços". A Agência Nacional de Vigilância Sanitária (ANVISA) concedeu à indústria o prazo de 12 meses para adequação dos rótulos à RDC n 26/2015. Este estudo tem como objetivo analisar os rótulos de AUP consumidos por crianças menores de cinco anos, quanto à adequação à Resolução n² 26, de 2 de julho de 2015, identificando informações sobre alimentos alergênicos.

\section{MÉTODOS}

Foram utilizados dados coletados sobre as práticas alimentares de crianças participantes de um estudo seccional, realizado no período de junho a dezembro de 2014, no Rio de Janeiro. A amostra do estudo maior foi probabilística, com seleção em dois estágios (estratificação das Unidades Básicas de Saúde - UBS por porte e sorteio de crianças nas listagens de cadastro das UBS) e foi composta por crianças com idade entre seis e 59 meses, assistidas em 33 UBS do Sistema Único de Saúde (SUS), distribuídas por bairros de diferentes zonas da cidade. O tamanho amostral foi calculado de forma a permitir análises estratificadas por faixa etária ( 6 a 23,$9 ; \geq 24$ meses) para os desfechos principais do estudo maior (prevalência de anemia e deficiência de vitamina A). Crianças com doença falciforme ou outras hemoglobinopatias não foram incluídas.

A partir dos dados de consumo alimentar obtido por meio de recordatório de 24 horas, foram identificados os alimentos ultraprocessados consumidos. Foi feita uma pesquisa de mercado para registro fotográfico dos rótulos desses alimentos. As informações coletadas nos rótulos dos alimentos ou no site dos fabricantes (quando os produtos não foram encontrados no mercado) foram: marca, ingredientes, nomes genéricos e alegações dos alimentos relatados. Os dados coletados foram digitados no Software Excel Microsoft $®$ (versão 2010).

Foram encontrados 303 AUP, que foram agrupados de acordo com suas características sensoriais e usos na alimentação, sendo divididos em grupos $(n=19){ }^{13}$

Os rótulos dos AUP fotografados foram comparados com a RDC n²6/2015, legislação específica para controle de alergênicos. Não foram incluídos na análise os alimentos em que não foi possível o acesso à lista de ingredientes, bem como as fórmulas infantis e os suplementos alimentares, por terem fins específicos e serem indicados para situações que requerem intervenções clínicas. É importante dizer que o estudo retrata um momento de 
transição da implantação das novas regras, uma vez que a coleta de dados foi realizada entre novembro e dezembro de 2015, e o prazo da ANVISA para a adequação dos rótulos era julho de 2016.

Os artigos sexto e sétimo da RDC n²6/2015 dispõem sobre como os alimentos que causam alergias alimentares devem obrigatoriamente ser apresentados nos rótulos, segundo requisitos estabelecidos (quadro 1).

Quadro 1. Inscrições que devem constar nos rótulos de produtos alimentícios com ingredientes alergênicos segundo a Resolução RDC n² 26 de 2015.

\begin{tabular}{|c|c|c|c|c|}
\hline \multirow{8}{*}{$\begin{array}{c}\text { Para os alimentos, } \\
\text { ingredientes, aditivos } \\
\text { alimentares e coadjuvantes } \\
\text { de tecnologia que } \\
\text { contenham ou sejam } \\
\text { derivados de } \\
\text { alergênicos }\end{array}$} & \multicolumn{2}{|c|}{ Contém } & Contém derivados & Pode conter \\
\hline & $\begin{array}{l}\text { nomes comuns } \\
\text { dos alimentos } \\
\text { que causam } \\
\text { alergias } \\
\text { alimentares }\end{array}$ & $\begin{array}{l}\text { nomes comuns } \\
\text { dos alimentos } \\
\text { que causam } \\
\text { alergias } \\
\text { alimentares e } \\
\text { derivados }\end{array}$ & $\begin{array}{l}\text { nomes comuns } \\
\text { dos alimentos } \\
\text { que causam } \\
\text { alergias } \\
\text { alimentares }\end{array}$ & $\begin{array}{l}\text { nomes comuns } \\
\text { dos alimentos } \\
\text { que causam } \\
\text { alergias } \\
\text { alimentares }\end{array}$ \\
\hline & \multicolumn{4}{|c|}{ Caixa alta } \\
\hline & \multicolumn{4}{|c|}{ Negrito } \\
\hline & \multicolumn{4}{|c|}{ Cor contrastante com o fundo do rótulo } \\
\hline & \multicolumn{4}{|c|}{$\begin{array}{c}\text { Altura mínima de } 2 \text { mm (não é permitida altura inferior à da letra utilizada na } \\
\text { lista de ingredientes) }\end{array}$} \\
\hline & \multicolumn{4}{|c|}{ Informações em local visível mesmo depois da abertura da embalagem } \\
\hline & \multicolumn{4}{|c|}{$\begin{array}{l}\text { Embalagens com painel textual igual ou inferiores a } 100 \mathrm{~cm}^{2} \text { a altura mínima } \\
\text { dos caracteres é de } 1 \mathrm{~mm}\end{array}$} \\
\hline
\end{tabular}

Para análise de dados, foram considerados todos os alimentos alergênicos e seus derivados mencionados na RDC n²6/2015, assim como as orientações das informações que deveriam estar presentes no rótulo, segundo esta regulamentação.

O estudo que deu origem ao banco de dados analisado foi aprovado pelo Comitê de Ética em Pesquisa com Seres Humanos da Secretaria Municipal de Saúde do Rio de Janeiro $\left(n^{\circ}\right.$ 93/2013). Os responsáveis pelas crianças estudadas concordaram com a participação e assinaram um Termo de Consentimento Livre e Esclarecido (TCLE).

\section{RESULTADOS}

Os 303 alimentos ultraprocessados estudados, de diferentes marcas e sabores, foram divididos em 19 grupos: bebidas açucaradas $(n=77)$, biscoitos $(n=53)$, doces e guloseimas $(n=47)$, 
iogurtes ultraprocessados e bebidas lácteas ( $n=34)$, farinhas espessantes $(n=14)$, salgadinhos e chips ( $n=14)$, temperos prontos em cubos e em pó ( $n=11)$, bolos industrializados ( $n=7)$, requeijão e queijos ultraprocessados ( $n=7)$, suco concentrado $(n=7)$, carnes industrializadas e embutidos $(n=6)$, pães $(n=6)$, achocolatados e saborizantes à base de morango $(n=4)$, cereal matinal $(n=4)$, molhos industrializados $(n=4)$, margarinas $(n=3)$, macarrões instantâneos $(n=2)$, pipocas industrializadas ( $\mathrm{n}=2)$ e farofas industrializadas ( $\mathrm{n}=1$ ) (tabela 1).

Tabela 1. Frequência de ingredientes alergênicos presentes na lista de ingredientes e frequência da presença de mensagem prevista na legislação nos rótulos de alimentos ultraprocessados consumidos por crianças, entre seis meses e cinco anos, atendidas no SUS no município do Rio de Janeiro, 2015.

\begin{tabular}{|c|c|c|c|c|c|}
\hline \multirow{2}{*}{$\begin{array}{l}\text { Grupo de alimentos } \\
\text { ultraprocessados (número de } \\
\text { produtos analisados) }\end{array}$} & \multicolumn{3}{|c|}{$\begin{array}{l}\text { Frequência de ingredientes alergênicos presentes } \\
\text { nos rótulos }\end{array}$} & \multicolumn{2}{|c|}{$\begin{array}{l}\text { Frequência da presença de mensagem } \\
\text { prevista na legislação }\end{array}$} \\
\hline & & $n$ & $\%$ & $\mathrm{n}$ & $\%$ \\
\hline \multirow{2}{*}{$\begin{array}{l}\text { Bebidas açucaradas } \\
(n=77)\end{array}$} & Leite & 1 & 1,29 & 1 & 100,00 \\
\hline & Soja & 13 & 16,88 & 4 & 30,76 \\
\hline \multirow{12}{*}{$\begin{array}{l}\text { Biscoitos } \\
(\mathrm{n}=53)\end{array}$} & Leite & 33 & 62,20 & 21 & 63,63 \\
\hline & Soja & 14 & 26,41 & 10 & 71,42 \\
\hline & Amendoim & 5 & 9,43 & 5 & 100,00 \\
\hline & Ovos & 2 & 3,77 & 2 & 100,00 \\
\hline & Centeio & 3 & 5,66 & 3 & 100,00 \\
\hline & Amêndoa & 2 & 3,77 & 2 & 100,00 \\
\hline & Nozes & 3 & 5,66 & 3 & 100,00 \\
\hline & Avelã & 2 & 3,77 & 2 & 100,00 \\
\hline & Aveia & 2 & 3,77 & 2 & 100,00 \\
\hline & Cevada & 7 & 13,20 & 6 & 85,71 \\
\hline & Trigo & 52 & 98,10 & 9 & 17,30 \\
\hline & Castanha & 3 & 5,60 & 3 & 100,00 \\
\hline \multirow{8}{*}{$\begin{array}{l}\text { Doces e guloseimas } \\
\qquad(n=47)\end{array}$} & Leite & 15 & 31,91 & 4 & 26,66 \\
\hline & Soja & 21 & 44,68 & 10 & 21,27 \\
\hline & Amêndoa & 3 & 6,38 & 3 & 100,00 \\
\hline & Avelã & 4 & 8,51 & 4 & 100,00 \\
\hline & Nozes & 2 & 4,52 & 2 & 100,00 \\
\hline & Amendoim & 6 & 12,76 & 3 & 50,00 \\
\hline & Castanha & 4 & 8,50 & 4 & 100,00 \\
\hline & Soja & 2 & 4,25 & 2 & 100,00 \\
\hline \multirow{7}{*}{$\begin{array}{l}\text { logurtes ultraprocessados e } \\
\text { bebidas lácteas } \\
\qquad(\mathrm{n}=34)\end{array}$} & Leite & 34 & 100,00 & 7 & 20,58 \\
\hline & Soja & 1 & 2,94 & 1 & 100,00 \\
\hline & Aveia & 1 & 2,94 & 0 & 0 \\
\hline & Amendoim & 1 & 2,94 & 1 & 100,00 \\
\hline & Cevada & 1 & 2,94 & 1 & 100,00 \\
\hline & Trigo & 2 & 5,88 & 0 & 0 \\
\hline & Castanha & 1 & 2,94 & 1 & 100,00 \\
\hline \multirow{4}{*}{$\begin{array}{l}\text { Farinhas espessantes } \\
\qquad(\mathrm{n}=14)\end{array}$} & Leite & 10 & 71,42 & 9 & 90,00 \\
\hline & Aveia & 3 & 21,42 & 0 & 0 \\
\hline & Soja & 2 & 14,28 & 2 & 100,00 \\
\hline & Trigo & 3 & 21,42 & 0 & 0 \\
\hline
\end{tabular}


Tabela 1. Frequência de ingredientes alergênicos presentes na lista de ingredientes e frequência da presença de mensagem prevista na legislação nos rótulos de alimentos ultraprocessados consumidos por crianças, entre seis meses e cinco anos, atendidas no SUS no município do Rio de Janeiro, 2015. (cont.)

\begin{tabular}{|c|c|c|c|c|c|}
\hline \multirow{2}{*}{$\begin{array}{l}\text { Grupo de alimentos } \\
\text { ultraprocessados (número de } \\
\text { produtos analisados) }\end{array}$} & \multicolumn{3}{|c|}{$\begin{array}{l}\text { Frequência de ingredientes alergênicos presentes } \\
\text { nos rótulos }\end{array}$} & \multicolumn{2}{|c|}{$\begin{array}{l}\text { Frequência da presença de mensagem } \\
\text { prevista na legislação }\end{array}$} \\
\hline & & $n$ & $\%$ & $n$ & $\%$ \\
\hline \multirow{3}{*}{$\begin{array}{l}\text { Salgadinhos e chips } \\
\qquad(n=14)\end{array}$} & Leite & 6 & 42,85 & 2 & 16,60 \\
\hline & Trigo & 4 & 28,57 & 0 & 0 \\
\hline & Soja & 6 & 42,85 & 6 & 100,00 \\
\hline \multirow{2}{*}{$\begin{array}{c}\text { Temperos prontos em cubo e } \\
\text { em pó } \\
(\mathrm{n}=11)\end{array}$} & & & & & \\
\hline & Soja & 2 & 18,18 & 2 & 100 \\
\hline \multirow{9}{*}{$\begin{array}{l}\text { Bolos industrializados } \\
\qquad(n=7)\end{array}$} & Leite & 2 & 28,57 & 2 & 100,00 \\
\hline & Soja & 3 & 42,85 & 3 & 100,00 \\
\hline & Amendoim & 1 & 14,28 & 1 & 100,00 \\
\hline & Ovos & 5 & 71,42 & 1 & 20,00 \\
\hline & Trigo & 7 & 100,00 & 2 & 28,57 \\
\hline & Amêndoa & 1 & 14,28 & 1 & 100,00 \\
\hline & Avelã & 1 & 14,28 & 1 & 100,00 \\
\hline & Cevada & 1 & 14,28 & 1 & 100,00 \\
\hline & Castanha & 1 & 14,28 & 1 & 100,00 \\
\hline \multirow{3}{*}{$\begin{array}{c}\text { Requeijão e queijos } \\
\text { ultraprocessados } \\
(n=7)\end{array}$} & Leite & 7 & 100,00 & 5 & 71,42 \\
\hline & Soja & 2 & 28,57 & 2 & 100,00 \\
\hline & Ovos & 1 & 14,28 & 1 & 100,00 \\
\hline $\begin{array}{l}\text { Sucos concentrados } \\
(\mathrm{n}=7)\end{array}$ & - & - & - & - & - \\
\hline \multirow{3}{*}{$\begin{array}{c}\text { Carnes industrializadas e } \\
\text { embutidos } \\
(n=6)\end{array}$} & Leite & 1 & 16,60 & 0 & 0 \\
\hline & Soja & 4 & 66,60 & 0 & 0 \\
\hline & Trigo & 4 & 66,60 & 0 & 0 \\
\hline \multirow{3}{*}{$\begin{array}{l}\text { Pães } \\
(n=6)\end{array}$} & Leite & 4 & 66,60 & 0 & 0 \\
\hline & Ovos & 1 & 16,66 & 0 & 0 \\
\hline & Trigo & 6 & 100,00 & 0 & 0 \\
\hline \multirow{5}{*}{$\begin{array}{l}\text { Achocolatados e saborizantes } \\
\text { à base de morango } \\
\qquad(n=4)\end{array}$} & Leite & 2 & 50,00 & 1 & 50,00 \\
\hline & Cevada & 2 & 50,00 & 0 & 0 \\
\hline & Aveia & 1 & 25,00 & 0 & 0 \\
\hline & Soja & 4 & 100,00 & 1 & 25,00 \\
\hline & Trigo & 2 & 50,00 & 1 & 50,00 \\
\hline \multirow{3}{*}{$\begin{array}{l}\text { Cereal matinal } \\
\qquad(\mathrm{n}=4)\end{array}$} & Leite & 3 & 75,00 & 3 & 100,00 \\
\hline & Amêndoa & 2 & 50,00 & 2 & 100,00 \\
\hline & Soja & 1 & 25,00 & 1 & 100,00 \\
\hline $\begin{array}{l}\text { Molhos industrializados } \\
(\mathrm{n}=4)\end{array}$ & - & - & - & - & - \\
\hline \multirow{2}{*}{$\begin{array}{l}\text { Margarinas } \\
(\mathrm{n}=3)\end{array}$} & Leite & 2 & 66,60 & 2 & 100,00 \\
\hline & Soja & 2 & 66,60 & 2 & 100,00 \\
\hline \multirow{4}{*}{$\begin{array}{l}\text { Macarrões instantâneos } \\
\qquad(\mathrm{n}=2)\end{array}$} & Leite & 1 & 50,00 & 1 & 100,00 \\
\hline & Soja & 2 & 100,00 & 2 & 100,00 \\
\hline & Ovos & 1 & 50,00 & 1 & 100,00 \\
\hline & Trigo & 1 & 50,00 & 1 & 100,00 \\
\hline
\end{tabular}


Tabela 1. Frequência de ingredientes alergênicos presentes na lista de ingredientes e frequência da presença de mensagem prevista na legislação nos rótulos de alimentos ultraprocessados consumidos por crianças, entre seis meses e cinco anos, atendidas no SUS no município do Rio de Janeiro, 2015. (cont.)

\begin{tabular}{|c|c|c|c|c|}
\hline \multirow{2}{*}{$\begin{array}{l}\text { Grupo de alimentos } \\
\text { ultraprocessados (número de } \\
\text { produtos analisados) }\end{array}$} & \multicolumn{2}{|c|}{$\begin{array}{l}\text { Frequência de ingredientes alergênicos presentes } \\
\text { nos rótulos }\end{array}$} & \multicolumn{2}{|c|}{$\begin{array}{c}\text { Frequência da presença de mensagem } \\
\text { prevista na legislação }\end{array}$} \\
\hline & $n$ & $\%$ & $n$ & $\%$ \\
\hline \multirow{2}{*}{$\begin{array}{l}\text { Pipocas industrializadas } \\
(\mathrm{n}=2)\end{array}$} & Leite & 50,00 & 1 & 100,00 \\
\hline & Soja & 50,00 & 1 & 100,00 \\
\hline $\begin{array}{l}\text { Farofas industrializadas } \\
\qquad(\mathrm{n}=1)\end{array}$ & Soja & 100,00 & 1 & 100,00 \\
\hline Total $(n=303)$ & 357 & - & 179 & - \\
\hline
\end{tabular}

(-) Não continha ingrediente alergênico

A tabela 1 mostra o número de ingredientes alergênicos por grupo de alimentos. Os grupos biscoitos, bolos industrializados, doces e guloseimas, iogurtes processados e bebidas lácteas e achocolatados e saborizantes à base de morango foram os que apresentaram o maior número de ingredientes alergênicos com relevância para a saúde pública. A quantidade de diferentes tipos de ingredientes alergênicos encontrados por grupo foi: em biscoitos, 12; em bolos industrializados, 9; em doces e guloseimas, 8; em iogurtes ultraprocessados e bebidas lácteas, 7; e em achocolatados e saborizantes à base de morango, 5.

Do total de alimentos alergênicos mencionados na RDC n²6/2015, foram encontrados 12 ingredientes alergênicos nos AUP estudados. Dois grupos de AUP não apresentaram nenhum alimento com ingredientes alergênicos: o grupo de sucos concentrados e o de molhos industrializados. Os 17 grupos de AUP restantes tinham um ou mais alimentos com ingredientes alergênicos. Soja e derivados estavam presentes em alimentos de 17 grupos; leite e derivados, na lista de 16 grupos; trigo e derivados, na lista de sete grupos; ovos, na lista de cinco grupos; amendoim, castanha, amêndoa, cevada e aveia, na lista de quatro grupos; avelã, na lista de três grupos; nozes, na lista de dois grupos e centeio, na lista de um grupo.

Entre os AUP que possuíam ingredientes alergênicos na lista de ingredientes, alguns grupos tinham proporção maior de produtos com a presença desses alergênicos em sua composição. Os grupos que apresentaram alimentos que continham soja foram: macarrões instantâneos (100\%), farofas industrializadas (100\%), achocolatados e saborizantes à base de morango (100\%), carnes industrializadas e embutidas (66,6\%), margarinas $(66,6 \%)$ e pipocas industrializadas (50\%). Os maiores percentuais de alimentos que continham leite foram dos seguintes grupos: iogurtes ultraprocessados e bebidas lácteas (100\%), requeijão e queijos ultraprocessados (100\%), cereal matinal (75\%), farinhas espessantes (71,42\%), margarinas 
(66,6\%), pães (66,6), biscoitos (62,2\%), achocolatados e saborizantes à base de morango (50\%), pipocas industrializadas (50\%) e macarrões instantâneos (50\%). Os grupos com maiores percentuais de alimentos com trigo foram: pães (100\%), bolos industrializados (100\%), biscoitos (98,1\%), carnes industrializadas e embutidos $(66,6 \%)$ e macarrões instantâneos (50\%). Com relação ao ovo, apresentaram maiores percentuais os grupos bolos industrializados $(71,2 \%)$ e macarrões instantâneos (50\%). Os grupos que apresentaram maiores percentuais de produtos com castanha foram: bolos industrializados $(14,28 \%)$, doces e guloseimas $(8,5 \%)$ e biscoitos $(5,6 \%)$. No que se refere ao amendoim, apresentaram os maiores percentuais os grupos: bolos industrializados (14,28\%), doces e guloseimas (12,76\%) e biscoitos (9,43\%). Quanto à presença de cevada, os grupos foram: achocolatados e saborizantes à base de morango (50\%), biscoitos $(13,2 \%)$ e bolos industrializados (14,28\%) apresentaram os maiores percentuais. Com relação à presença de aveia, os grupos foram: achocolatados e saborizantes à base de morango (25\%) e farinhas espessantes $(21,42 \%)$. Nozes foram listadas somente em alimentos de dois grupos, tendo o maior percentual o grupo dos biscoitos (5,66\%); avelã esteve presente no grupo dos bolos industrializados (14,28\%) e no de doces e guloseimas (8,51\%); e amêndoa no grupo de cereal matinal (50\%). Com relação ao centeio, somente o grupo dos biscoitos (5,66\%) apresentou este ingrediente (tabela 1).

Nos rótulos analisados, a apresentação da mensagem obrigatória para alérgicos foi encontrada na minoria dos alimentos em que os ingredientes alergênicos estavam presentes (33,1\%). Nos grupos carnes industrializadas e embutidos e grupo dos pães, não foram encontradas mensagens obrigatórias alertando a presença de nenhum ingrediente alergênico. Foram parcialmente encontradas advertências quanto à presença de alergênicos nos rótulos de alimentos dos grupos bebidas açucaradas ( 5 de 14, ou seja, das 14 vezes em que os ingredientes alergênicos estavam presentes na lista de ingredientes, apenas cinco apresentaram a mensagem obrigatória), biscoitos (68 de 128), doces e guloseimas (32 de 57), iogurtes ultraprocessados e bebidas lácteas (11 de 41), farinhas espessantes (11 de 18), salgadinhos e chips (8 de 16), bolos industrializados (13 de 22), requeijão e queijos ultraprocessados (8 de 10), achocolatados e saborizantes à base de morango (3 de 11). Nos grupos temperos prontos em cubo e em pó, cereal matinal, margarinas, macarrões instantâneos, pipocas industrializadas e farofas industrializadas, foram encontradas mensagens obrigatórias sobre a presença de alergênicos em todos os rótulos analisados. Os grupos molhos industrializados e sucos concentrados não apresentaram ingredientes com potencial alergênico em suas composições (tabela 1). 
Também foram identificados os derivados dos ingredientes alergênicos dos alimentos consumidos pelas crianças. Os grupos em que os ingredientes derivados aparecem em maior quantidade são os grupos biscoitos, doces e guloseimas, iogurtes ultraprocessados e bebidas lácteas e salgadinhos e chips. No grupo dos biscoitos, a presença de derivados na lista de ingredientes foi declarada 103 vezes, porém somente em 33 alimentos havia a mensagem obrigatória nos rótulos. Em doces e guloseimas, foi declarada 29 vezes a presença de derivados, dos quais somente 12 traziam mensagem obrigatória nos rótulos. No grupo iogurtes ultraprocessados e bebidas lácteas, a presença de derivados na lista de ingredientes foi declarada 29 vezes, porém apenas nove foram declarados nos rótulos. Em salgadinhos e chips, a presença de derivados de alimentos alergênicos foi declarada 22 vezes, dos quais apenas dois apresentavam a declaração no rótulo (tabela 2).

Tabela 2. Frequência de derivados de alimentos alergênicos e frequência da presença da mensagem correspondente prevista na legislação nos rótulos de alimentos ultraprocessados consumidos por crianças entre seis meses e cinco anos atendidas na rede SUS no município do Rio de Janeiro em 2015

\begin{tabular}{|c|c|c|c|c|c|}
\hline \multirow[t]{2}{*}{$\begin{array}{l}\text { Grupo de alimentos ultraprocessados } \\
\text { (número de produtos analisados) }\end{array}$} & \multicolumn{2}{|c|}{$\begin{array}{l}\text { Frequência de derivados de alimentos } \\
\text { alergênicos presentes nos rótulos }\end{array}$} & \multicolumn{3}{|c|}{$\begin{array}{c}\text { Frequência da presença de } \\
\text { mensagem prevista na legislação }\end{array}$} \\
\hline & & $n$ & $\%$ & $\mathrm{n}$ & $\%$ \\
\hline & Lecitina de soja & 2 & 2,59 & 0 & 0 \\
\hline \multirow{3}{*}{$\begin{array}{l}\text { Bebidas açucaradas } \\
\qquad(\mathrm{n}=77)\end{array}$} & Extrato de soja & 4 & 5,19 & 0 & 0 \\
\hline & Grãos de soja & 3 & 3,89 & 1 & 33,33 \\
\hline & Proteína de soja & 3 & 3,89 & 0 & 0 \\
\hline \multirow{3}{*}{$\begin{array}{l}\text { Biscoitos } \\
(n=53)\end{array}$} & Lecitina de soja & 38 & 71,69 & 15 & 39,47 \\
\hline & Soro do leite & 13 & 24,50 & 6 & 46,15 \\
\hline & Farinha de trigo & 52 & 98,11 & 12 & 23,07 \\
\hline \multirow{5}{*}{$\begin{array}{l}\text { Doces e guloseimas } \\
\qquad(n=47)\end{array}$} & Lecitina de soja & 21 & 44,68 & 10 & 47,61 \\
\hline & Soro do leite & 4 & 8,51 & 1 & 25,00 \\
\hline & Creme de leite & 1 & 2,12 & 1 & 100,00 \\
\hline & Farinha de trigo & 2 & 4,25 & 0 & 0 \\
\hline & Farinha de soja & 1 & 2,12 & 0 & 0 \\
\hline \multirow{6}{*}{$\begin{array}{l}\text { logurtes ultraprocessados e bebidas lácteas } \\
\qquad(\mathrm{n}=34)\end{array}$} & Lecitina de soja & 3 & 8,82 & 0 & 0 \\
\hline & Soro do leite & 12 & 35,29 & 4 & 33,33 \\
\hline & Proteína de soja & 3 & 8,82 & 1 & 33,33 \\
\hline & Proteína do leite & 2 & 5,58 & 1 & 2,94 \\
\hline & Farinha de trigo & 2 & 5,58 & 0 & 0 \\
\hline & Creme de leite & 7 & 20,58 & 3 & 42,85 \\
\hline
\end{tabular}


Tabela 2. Frequência de derivados de alimentos alergênicos e frequência da presença da mensagem correspondente prevista na legislação nos rótulos de alimentos ultraprocessados consumidos por crianças entre seis meses e cinco anos atendidas na rede SUS no município do Rio de Janeiro em 2015. (cont.)

\begin{tabular}{|c|c|c|c|c|c|}
\hline \multirow{2}{*}{$\begin{array}{c}\begin{array}{c}\text { Grupo de alimentos ultraprocessados } \\
\text { (número de produtos analisados) }\end{array} \\
\text { Farinhas espessantes } \\
(n=14)\end{array}$} & \multicolumn{2}{|c|}{$\begin{array}{l}\text { Frequência de derivados de alimentos } \\
\text { alergênicos presentes nos rótulos }\end{array}$} & \multicolumn{3}{|c|}{$\begin{array}{c}\text { Frequência da presença de } \\
\text { mensagem prevista na legislação }\end{array}$} \\
\hline & Farinha de trigo & 7 & 50,00 & 0 & 0 \\
\hline \multirow{5}{*}{$\begin{array}{l}\text { Salgadinhos e chips } \\
\qquad(\mathrm{n}=14)\end{array}$} & Lecitina de soja & 1 & 7,10 & 0 & 0 \\
\hline & Soro do leite & 8 & 57,10 & 1 & 12,50 \\
\hline & $\begin{array}{l}\text { Proteína hidrolisada de } \\
\text { trigo }\end{array}$ & 1 & 7,14 & 0 & 0 \\
\hline & Farinha de trigo & 3 & 21,42 & 0 & 0 \\
\hline & Queijo & 2 & 14,28 & 1 & 50,00 \\
\hline $\begin{array}{l}\text { Temperos prontos em cubo e em pó } \\
\qquad(n=11)\end{array}$ & - & - & - & - & - \\
\hline \multirow{3}{*}{$\begin{array}{l}\text { Bolos industrializados } \\
\qquad(\mathrm{n}=7)\end{array}$} & Farinha de trigo & 7 & 100,00 & 2 & 28,57 \\
\hline & Lecitina de soja & 1 & 14,28 & 0 & 0 \\
\hline & Farinha de soja & 1 & 14,28 & 0 & 0 \\
\hline \multirow{3}{*}{$\begin{array}{l}\text { Requeijão e queijos ultraprocessados } \\
\qquad(n=7)\end{array}$} & Soro do leite & 1 & 14,28 & 1 & 100,00 \\
\hline & Proteína de leite & 1 & 14,28 & 0 & 0 \\
\hline & Creme de leite & 5 & 71,42 & 2 & 40,00 \\
\hline $\begin{array}{c}\text { Sucos concentrados } \\
(\mathrm{n}=7)\end{array}$ & - & - & - & - & - \\
\hline \multirow{2}{*}{$\begin{array}{l}\text { Carnes industrializadas e embutidos } \\
\qquad(\mathrm{n}=6)\end{array}$} & Farinha de trigo & 4 & 50,00 & 0 & 0 \\
\hline & Proteína de soja & 6 & 100,00 & 0 & 0 \\
\hline \multirow{3}{*}{$\begin{array}{l}\text { Pães } \\
(n=6)\end{array}$} & Soro do leite & 2 & 33,33 & 0 & 0 \\
\hline & Farinha de trigo & 6 & 100,00 & 0 & 0 \\
\hline & Lecitina de soja & 4 & 66,66 & 1 & 25,00 \\
\hline \multirow{3}{*}{$\begin{array}{l}\text { Achocolatados e saborizantes à base de } \\
\text { morango } \\
(n=4)\end{array}$} & Lecitina de soja & 4 & 100,00 & 1 & 25,00 \\
\hline & Soro do leite & 2 & 50,00 & 1 & 50,00 \\
\hline & Farinha de trigo & 1 & 25,00 & 1 & 100,00 \\
\hline $\begin{array}{c}\text { Cereal matinal } \\
(\mathrm{n}=4)\end{array}$ & Soro do leite & 1 & 25,00 & 1 & 100,00 \\
\hline $\begin{array}{l}\text { Molhos industrializados } \\
(\mathrm{n}=4)\end{array}$ & - & - & & & \\
\hline \multirow{2}{*}{$\begin{array}{l}\text { Margarinas } \\
\qquad(\mathrm{n}=3)\end{array}$} & Lecitina de soja & 2 & 66,60 & 2 & 100,00 \\
\hline & Soro do leite & 1 & 33,30 & 1 & 100,00 \\
\hline $\begin{array}{l}\text { Macarrões instantâneos } \\
(\mathrm{n}=2)\end{array}$ & Farinha de trigo & 1 & 50,00 & 1 & 100,00 \\
\hline $\begin{array}{l}\text { Pipocas industrializadas } \\
\qquad(\mathrm{n}=2)\end{array}$ & Lecitina de soja & 1 & 50,00 & 1 & 100,00 \\
\hline
\end{tabular}


Tabela 2. Frequência de derivados de alimentos alergênicos e frequência da presença da mensagem correspondente prevista na legislação nos rótulos de alimentos ultraprocessados consumidos por crianças entre seis meses e cinco anos atendidas na rede SUS no município do Rio de Janeiro em 2015. (cont.)

\begin{tabular}{cccc}
\hline $\begin{array}{c}\text { Grupo de alimentos ultraprocessados } \\
\text { (número de produtos analisados) }\end{array}$ & $\begin{array}{c}\text { Frequência de derivados de alimentos } \\
\text { alergênicos presentes nos rótulos }\end{array}$ & $\begin{array}{c}\text { Frequência da presença de } \\
\text { mensagem prevista na legislação }\end{array}$ \\
\hline $\begin{array}{c}\text { Farofas industrializadas } \\
(\mathrm{n}=1)\end{array}$ & - & - & - \\
\hline Total & - & 246 & - \\
\hline
\end{tabular}

As informações "contém traços" e "pode conter" estavam presentes em aproximadamente 13 e 17\% dos rótulos, respectivamente. Ao verificar a presença da mensagem "contém traços", observou-se esta informação em bebidas açucaradas, biscoitos, doces e guloseimas, iogurtes ultraprocessados e bebidas lácteas, farinhas espessantes, salgadinhos e chips, bolos industrializados, achocolatados e saborizantes à base de morango e cereal matinal. 0 comunicado "pode conter" foi encontrado nos rótulos das bebidas açucaradas, biscoitos, doces e guloseimas, iogurtes ultraprocessados e bebidas lácteas, salgadinhos e chips, temperos prontos em cubos e em pó, bolos industrializados, requeijão e queijos ultraprocessados, achocolatados e saborizantes à base de morango, molhos industrializados, macarrões instantâneos, pipocas industrializadas e farofas industrializadas (tabela 3).

Tabela 3. Presença das mensagens "contém traços" e "pode conter", por grupos de alimentos ultraprocessados consumidos por crianças entre seis meses e cinco anos atendidas na rede SUS no município do Rio de Janeiro, em 2015.

\begin{tabular}{|c|c|c|c|c|}
\hline \multirow{2}{*}{$\begin{array}{l}\text { Grupo de alimentos ultraprocessados (número de } \\
\text { alimentos analisados) }\end{array}$} & \multicolumn{2}{|c|}{ "Contém traços" } & \multicolumn{2}{|c|}{ "Pode conter" } \\
\hline & $\mathrm{n}$ & $\%$ & $\mathrm{n}$ & $\%$ \\
\hline Bebidas açucaradas ( $n=77)$ & 2 & 2,59 & 1 & 1,29 \\
\hline Biscoitos $(n=53)$ & 9 & 16,98 & 12 & 26,41 \\
\hline Doces e guloseimas ( $n=47)$ & 7 & 14,89 & 9 & 19,14 \\
\hline logurtes ultraprocessados e bebidas lácteas $(n=34)$ & 2 & 5,88 & 11 & 29,41 \\
\hline Farinhas espessantes $(n=14)$ & 9 & 64,28 & - & - \\
\hline Salgadinhos e chips $(n=14)$ & 6 & 42,85 & 2 & 14,28 \\
\hline Temperos prontos em cubo e em pó ( $n=11)$ & - & - & 3 & 27,27 \\
\hline Bolos industrializados $(n=7)$ & 1 & 14,28 & 5 & 71,42 \\
\hline Suco concentrado $(n=7)$ & - & - & - & - \\
\hline Requeijão e queijos ultraprocessados ( $n=7$ ) & - & - & 2 & 28,57 \\
\hline Carnes industrializadas e embutidos $(n=6)$ & - & - & - & - \\
\hline Pães $(n=6)$ & - & - & - & - \\
\hline Achocolatados e saborizantes à base de morango $(n=4)$ & 1 & 25,00 & 1 & 25,00 \\
\hline Cereal matinal $(n=4)$ & 3 & 75,00 & - & - \\
\hline Molhos industrializados ( $\mathrm{n}=4)$ & - & - & 1 & 25,00 \\
\hline Margarinas $(n=3)$ & - & - & - & - \\
\hline
\end{tabular}


Tabela 3. Presença das mensagens "contém traços" e "pode conter", por grupos de alimentos ultraprocessados consumidos por crianças entre seis meses e cinco anos atendidas na rede SUS no município do Rio de Janeiro, em 2015. (cont.)

\begin{tabular}{lcccc}
\hline Grupo de alimentos ultraprocessados (número de & \multicolumn{2}{c}{ "Contém traços" } & \multicolumn{2}{c}{ "Pode conter" } \\
\cline { 2 - 5 } alimentos analisados) & $n$ & $\%$ & $n$ & $\%$ \\
\hline Macarrões instantâneos $(n=2)$ & - & - & 2 & 100 \\
Pipocas industrializadas $(n=2)$ & - & - & 2 & 100 \\
Farofas industrializadas $(n=1)$ & - & - & 1 & 100 \\
Total & 40 & - & 52 & - \\
\hline
\end{tabular}

(-) Não continha ingredientes alergênicos.

\section{DISCUSSÃO}

As crianças atendidas no SUS na cidade do Rio de Janeiro consumiram um largo repertório de alimentos ultraprocessados (97,9\%). ${ }^{14}$ Estudo realizado no Brasil, com amostra de indivíduos de 10 ou mais anos de idade, constatou que 21,5\% do consumo diário de energia são provenientes de alimentos ultraprocessados..$^{15}$ Outro estudo brasileiro, com crianças de 13 a 35 meses de idade, mostrou que 25,8\% do consumo diário de energia é obtido de AUP. ${ }^{16}$

A maioria (58,7\%) dos AUP analisados possuía algum tipo de ingrediente alergênico. Alguns $(33,1 \%)$ traziam a advertência quanto à presença de todos os alergênicos em sua lista de ingredientes. Mesmo antes de existir uma RDC que tratasse do assunto, isto já deveria estar claro nos rótulos, pois é direito do consumidor ter sua vida, saúde e segurança protegidas dos riscos no fornecimento de produtos considerados perigosos. ${ }^{17} \mathrm{~A}$ indústria deve se responsabilizar pelos produtos que comercializa, garantindo a informação dos ingredientes que fazem parte da composição deles.

Cabe informar que o estudo retrata um momento de transição, já que havia pelo menos quatro meses em que a legislação tinha sido publicada e as indústrias ainda estavam se adequando à legislação.

A necessidade de monitoramento das regulamentações sobre rotulagem de alimentos já foi apontada em outros estudos. Chaud et al., ${ }^{18}$ na cidade de São Paulo, mostraram que embora houvesse legislação para regulamentar a rotulagem de alimentos, muitos deles não traziam informações obrigatórias em seus rótulos. Isto nos faz questionar se, após o período de adequação previsto na RDC n²6/2015, as irregularidades encontradas no presente estudo não seriam mais identificadas.

Neste estudo, a maior parte dos alimentos alergênicos mencionados na RDC n²6/2015 foi encontrada no grupo dos biscoitos. Quando se pensa em crianças com AA, este achado se 
torna ainda mais relevante, pois os biscoitos fazem parte da rotina alimentar de crianças, como mostrou o estudo de Domingues, Miranda e Santana, ${ }^{19}$ o qual identificou que o consumo de biscoitos recheados ocorre numa frequência de duas vezes na semana. O consumo de macarrão instantâneo também requer atenção, uma vez que é oferecido precocemente às crianças, como mostrou o estudo de Toloni et al. ${ }^{20}$

O estudo de Rodrigues et al. ${ }^{21}$ encontrou conformidade com a legislação nos rótulos de iogurtes, bebidas lácteas e leite fermentado, quanto à presença de alergênicos. No entanto, diferentemente desses achados, os resultados deste estudo mostraram inconformidade na maior parte dos rótulos de iogurtes ultraprocessados e bebidas lácteas em relação à RDC $n^{\circ} 26 / 2015$.

A alimentação adequada integra o rol de direitos humanos, e cabe ao Estado programar políticas públicas necessárias para garanti-la. ${ }^{22}$ Em relação à legislação voltada para alimentos alergênicos, as inconformidades mostradas neste trabalho são preocupantes, pois colocam em risco um grupo vulnerável. Tais inconformidades podem fazer com que indivíduos que possuem alergias alimentares consumam estes alimentos e venham a ter reações alérgicas.

Além dos alimentos mencionados na RDC n² 26/2015, existem outros ingredientes que precisam de atenção, como é o caso daqueles obtidos a partir de derivados de alimentos capazes de causar alergias. Isto abrange alimentos, ingredientes, aditivos alimentares e coadjuvantes de tecnologia. ${ }^{3}$ Um dos derivados encontrados na análise dos AUP foi a lecitina de soja, que foi acrescentada na lista de ingredientes como um aditivo alimentar (emulsificante). Por mais que o uso de aditivos químicos seja permitido pela legislação, são crescentes as evidências de que não são inofensivos à saúde. Estudos recentes sugerem que o aumento do consumo de substâncias como emulsificantes, surfactantes, solventes orgânicos, transglutaminase microbiana e nanopartículas pode estar vinculado ao aumento da prevalência de doenças autoimunes nas últimas décadas. A hipótese baseia-se no fato de que essas substâncias danificam os mecanismos de proteção intestinal contra antígenos externos, aumentando o risco de doenças imunológicas. ${ }^{23}$ Além disso, um estudo experimental observou que camundongos que receberam baixas concentrações de emulsificantes apresentaram alterações da microbiota intestinal que levaram à inflamação, ganho de peso e síndrome metabólica. ${ }^{24}$

Vale ressaltar que, neste caso, a presença de aditivos é intencional. Portanto, de acordo com a RDC n²6/2015, deve ser declarada a presença de aditivos como se fosse qualquer outro alimento alergênico, ainda que sob a alegação de ser utilizado na indústria em pequenas quantidades. O controle de alergênico possui caráter preventivo, e a indústria deve ter a 
consciência de que pequenas quantidades de alergênicos alimentares podem ser o bastante para gerar reações adversas em indivíduos com AA. ${ }^{3}$

A informação do risco de ter ocorrido contaminação cruzada durante o processamento do alimento pela indústria é essencial para indicar que o alimento não é seguro para indivíduos alérgicos. Nos rótulos dos AUP analisados, a informação "contém traços" estava presente em aproximadamente $13 \%$ dos alimentos. Estes rótulos estavam em desacordo com a RDC $n^{\circ} 26$, segundo a qual a mensagem que indica a possibilidade de ter algum ingrediente alergênico no alimento, ainda que de forma não intencional, deve ser "pode conter". 3 A informação "pode conter" estava presente em uma proporção um pouco maior.

A grande diversidade de grupos de AUP consumidos pelas crianças das Unidades Básicas de Saúde (UBS) aponta que o padrão alimentar destas inclui alimentos com quantidades excessivas de açúcar, sódio, gordura saturada, corantes e ingredientes alergênicos, além de baixo teor de fibras alimentares e vitaminas. ${ }^{9} \mathrm{O}$ alto consumo de AUP produz impacto negativo na saúde de crianças e implicações no desenvolvimento infantil. Os alimentos in natura e minimamente processados possuem teor de micronutrientes mais alto que os ultraprocessados. Dessa forma, o surgimento de carências de vitaminas e minerais está associado ao aumento do consumo de AUP e diminuição de alimentos in natura e minimamente processados. ${ }^{15}$ As preparações caseiras à base de alimentos in natura e minimamente processados conferem maior variedade de alimentos. A presença de inúmeras marcas e sabores dos AUP pode dar falsa sensação de variedade, mas na verdade a variedade é de aditivos químicos. A maior parte dos produtos consumidos se baseia em preparações à base de açúcares e óleos (vindos do milho e da soja), trigo e leite. ${ }^{25}$

Os achados no presente trabalho demonstram a relevância da legislação que dispõe sobre os requisitos para rotulagem obrigatória dos principais alimentos que causam alergias alimentares. A leitura de rótulos por si só não garante que o alimento esteja isento de substâncias alergênicas. É necessário que as informações a respeito dos ingredientes que compõem os alimentos sejam especificadas de forma clara, indicando, inclusive, derivados de substâncias alergênicas que são também capazes de desencadear reações alérgicas.

Uma das limitações do estudo foi que a RDC n²6 foi sancionada em julho de 2015, e a coleta de dados foi realizada entre novembro e dezembro de 2015. Como a ANVISA permitiu o prazo de um ano para adequação dos rótulos, ou seja, até julho de 2016, os dados encontrados são de um período de transição. No entanto, estudo de Miranda e Gama, que analisou rótulos de produtos comercializados em data posterior ao prazo de adequação de mensagem obrigatória de presença de alergênicos, demonstrou que 31,7\% dos rótulos permaneciam 
inadequados em relação à RDC n²6/2015. Esses dados corroboram os achados do presente estudo, que se propôs a demonstrar a dificuldade de adequação das indústrias e a necessidade de monitoramento diante dos achados inadequados, mesmo após a implementação de legislação específica para ingredientes alergênicos. ${ }^{26}$

\section{CONCLUSÃO}

A RDC n² 26/2015 é de grande importância, por auxiliar na garantia do direito à informação, possibilitando que as informações claras e padronizadas nos rótulos dos alimentos sejam utilizadas como ferramenta para o cuidado da alimentação de pessoas vivendo com alergia alimentar. Os achados deste estudo sugerem que, no momento da transição desta RDC, a maior parte dos rótulos não estava regular.

Destaca-se a necessidade de novos estudos que auxiliem no monitoramento dessa legislação. Apesar de a RDC n 26/2015 ser um bom instrumento de apoio, é fundamental que ela traga informações mais precisas para os alérgicos, como a quais derivados de alimentos alergênicos podem causar reações alérgicas.

A ausência de clareza e padronização dessas informações pode confundir os consumidores, implicando maior exposição a alimentos alergênicos. Saber ler os rótulos auxilia a minimizar os riscos relacionados ao consumo de ultraprocessados por alérgicos. Contudo, nossos achados também reforçam que o consumo de AUP representa um perigo à saúde de pessoas vivendo com alergia alimentar. O caminho seguro para evitar riscos à saúde desses indivíduos é a diminuição do consumo de alimentos ultraprocessados e a valorização da culinária doméstica, considerando o uso preferencialmente de alimentos in natura e minimamente processados.

\section{REFERÊNCIAS}

1. Sicherer SH, Sampson HA. Food allergy: a review and update on epidemiology, pathogenesis, diagnosis, prevention, and management. J Allergy Clin Immunol. 2018; 141:41-58.

2. Solé D, Silva LR, Cocco RR, Ferreira CT, Sarni RO, Oliveira LC, et al. Consenso Brasileiro sobre Alergia Alimentar: Etiopatogenia, clínica e diagnóstico. Documento conjunto elaborado pela Sociedade Brasileira de Pediatria e Associação Brasileira de Alergia e Imunologia. 2018; 2(1): 39-82.

3. Brasil. Agência Nacional de Vigilância Sanitária. Guia sobre programa de controle de alergênicos. Versão 1. Brasília, DF: ANVISA; 2016. 
4. Sociedade Brasileira de Pediatria. Manual de Orientação. Rio de Janeiro: Departamento de Nutrologia; 2012.

5. Rotenberg S, Vargas S. Práticas alimentares e o cuidado da saúde: da alimentação da criança à alimentação da família. Rev. Bras. Saúde Matern. Infant. 2004; 4(1): 85-94.

6. Freitas MCS, Pena PGL, Fontes GAV, Silva DO. Hábitos alimentares e o sentido de comer. In: DiezGarcia RW, Cervato-Mancuso AM. Mudanças alimentares e educação nutricional. Rio de Janeiro: Guanabara Koogan; 2013. p. 35-42.

7. Brasil. Ministério da Saúde. Pesquisa Nacional de Demografia e Saúde da Criança e da Mulher, PNDS 2006. Brasília DF, 2009. Acesso em: 2016 nov 21. Disponível em:<http://bvsms.saude.gov.br/bvs/pnds/index.php>.

8. Instituto Brasileiro de Geografia e Estatística. Pesquisa Nacional de Saúde, 2013. Percepção do Estado de Saúde, estilos de vida e doenças crônicas. 2014. Acesso em: 2016 nov 21. Disponível em: $<$ https://loja.ibge.gov.br/pesquisa-nacional-de-saude-2013-percepc-o-do-estado-de-saude-estilosde-vida-e-doencas-cronicas.html>.

9. Brasil. Ministério da Saúde. Guia alimentar para a população brasileira. 2. ed. Brasília: Ministério da Saúde; 2014.

10. Criolo LFR. Monitoramento e avaliação da regulamentação sobre rotulagem de alimentos alergênicos no Brasil: proposição de indicadores e métricas. 2016. 116 f. Dissertação (Mestrado em Metrologia) Pontifícia Universidade Católica do Rio de Janeiro, Rio de Janeiro; 2016.

11. Brasil. Ministério da Saúde. Secretaria de Atenção à Saúde. Departamento de Atenção Básica. Política Nacional de Atenção Básica. Brasília: Ministério da Saúde, 2012.

12. Brasil. Agência Nacional de Vigilância Sanitária. Resolução $n^{\circ} 26$, de 2 de julho de 2015. Dispõe sobre os requisitos para rotulagem obrigatória dos principais alimentos que causam alergias alimentares. Diário Oficial da União, 3 jul. 2015.

13. Avelar CO. Caracterização dos alimentos ultraprocessados consumidos por crianças de seis meses a cinco anos de idade usuárias de unidades básicas de saúde (UBS) no município do Rio de Janeiro. Rio de Janeiro. Dissertação (Mestrado em Alimentação, Nutrição e Saúde). Universidade do Estado do Rio de Janeiro; 2017.

14. Oliveira JM. Práticas alimentares de crianças menores de dois anos atendidas pelo Sistema Único de Saúde na cidade do Rio de Janeiro: validação de instrumento e padrões alimentares. Rio de Janeiro. Tese (Doutorado em Alimentação, Nutrição e Saúde). Universidade do Estado do Rio de Janeiro; 2018.

15. Louzada MLC, Baraldi LG, Steele EM, Martins APB, Canella DS, Moubarac JC, et al. Preventive Medicine. 2015; 81: 9-1. 
16. Batalha MA, França AKTC, Conceição SIO, Santos AM, Silva FS, Padilha LL, et al. Consumo de alimentos processados e ultraprocessados e fatores associados em crianças entre 13 e 35 meses de idade. Cad. Saúde Pública. 2017; 33(11): 1-16.

17. Brasil. Lei $n^{\circ}$. 8.078 - 11 de setembro de 1990. Código de defesa do consumidor. São Paulo: Enciclopédia Britânica do Brasil; 1991.

18. Chaud DMA, Braga MM, Abreu ES. Avaliação dos rótulos de alimentos diet e light comercializados em um empório da cidade de São Paulo (SP). Rev. Simbio-Logias. 2011; 4(6).

19. Domingues AG, Miranda AS, Santana RF. Consumo de alimentos industrializados em crianças de unidade escolar pública da cidade de Jacaraci-BA. C\&D-Revista Eletrônica da Fainor. 2014; 7(2): 206218.

20. Toloni MHA. Introdução de alimentos industrializados e de alimentos de uso tradicional na dieta de crianças de creches públicas no município de São Paulo. Rev. Nutr., Campinas. 2011; 24(1): 61-70.

21. Rodrigues DEB, Santos CM, Machado SG. Avaliação da adequação da rotulagem de iogurte integral, bebida láctea e leite fermentado em relação â RDC N²6, de 02 de julho de 2015. [Anais do VI Seminário de Iniciação Científica do IFNMG; 2017; Minas Gerais, Brasil].

22. Chaddad MCC. Informação sobre a presença de alérgenos nos rótulos de alimentos: responsabilidade do estado na garantia dos direitos à saúde e à alimentação adequada da população com alergia alimentar. Demetra. 2014; 9(supl 1): 369-392.

23. Lerner A, Mathias T. Mudanças na permeabilidade da junção intestinal rígida associada a aditivos alimentares industriais explicam a crescente incidência de doenças autoimunes. Autoimmun Rev. 2015; 14(6): 479-489.

24. Chassaing B, Koren O, Goodrich JK, Poole AC, Srinivasan S, Lev RE, et al. Emulsificantes dietéticos causam impacto na microbiota intestinal do rato promovendo colite e síndrome metabólica. Nature. 2015; 519(7541): 92-96.

25. Ramos $P$, Júnior AOS. O açúcar e as transformações nos regimes alimentares. Cad. de Debate. 2001; 8:36-54.

26. Miranda CCS, Gama LLA. Inadequação da rotulagem de alergênicos: risco para indivíduos com hipersensibilidade alimentar. Demetra. 2018; 13(3):731-743.

\section{Colaboradoras}

Teixeira TV e dos Santos CB participaram do desenho de estudo, da análise e interpretação dos dados, da redação do artigo e da sua versão final. Damião JJ, Maldonado LA e Oliveira JM participaram da revisão e aprovação da versão final.

Conflito de interesses: as autoras declaram não haver conflito de interesses. 
(C) Alimentos ultraprocessados e crianças

Recebido: 04 de julho de 2019

Revisado: 10 de setembro de 2019

Aceito: 24 de setembro de 2019 